\title{
Endocytosis in plants: peculiarities and roles in the regulated trafficking of plant metal transporters
}

Rumen Ivanov ${ }^{1}$ and Grégory Vert ${ }^{*}$

${ }^{1}$ Institute of Botany, Heinrich-Heine University, D-40225 Düsseldorf, Germany

${ }^{2}$ Plant Science Research Laboratory (LRSV), UMR5546 CNRS/Université Toulouse 3, 24 chemin de Borde Rouge, 31320 Auzeville-Tolosane, France.

Correspondence: Gregory.Vert@1rsv.ups-tlse.fr 


\begin{abstract}
The removal of transmembrane proteins from the plasma membrane via endocytosis has emerged as powerful tool in the regulation of receptor signaling and molecule transport. In the last decade, IRON-REGULATED TRANSPORTER1 (IRT1) has been established as one of the key model proteins for studying endomembrane trafficking. The use of IRT1 and additional other metal transporters, have uncovered novel factors involved in plant endocytosis and facilitated a better understanding of the role of endocytosis in the fine balancing of plant metal homeostasis. In this review, we outline the specifics of plant endocytosis compared to what is known in yeast and mammals, and based on several examples, we demonstrate how studying metal transport has contributed to extending our knowledge of endocytic trafficking by shedding light on novel regulatory mechanisms and factors.
\end{abstract}

Keywords:

endocytosis, endocytic recycling, vacuolar degradation, ubiquitin, metal homeostasis, IRT1, NRAMP1

The plasma membrane represents the border limiting the free flow of material and a checkpoint for perception and integration of extracellular signals prior to signal transduction in living cells. The regulated exchange of molecules and signals across the plasma membrane is achieved with the help of transmembrane proteins such as, channels, transporters and receptors. Cellular import of metal ions is crucial for the function of plant cells, as most of these are essential nutrients required for the completion of the plant life cycle (Thomine and Vert 2013). At the same time, overaccumulation of essential micronutrients or the entry of undesired minerals, such as cadmium, can lead to toxicity, cell damage and death. Therefore, regulating 
the abundance and activity of metal transporters at the plasma membrane is a key regulatory step in plant mineral nutrition.

As a result of membrane vesicle exchange between the plasma membrane and the cytoplasmic endomembrane system, each cell type possesses a unique and dynamically-balanced set of transmembrane proteins, which determines the role of the cell in the development, and general homeostasis of the whole organism. Proteins are removed from the plasma membrane via the internalization of membrane patches into vesicles in a process called endocytosis (Figure 1) (Paez Valencia et al. 2016). Within endosomes, this material is then sorted for degradation or recycled back to the cell surface. Recycled proteins, together with newly synthesized ones, are secreted in the form of transport vesicles to replenish the functional protein population to the plasma membrane (Figure 1). Below, we will review the organization of plant endocytosis and endomembrane trafficking, as characterized in the model plant Arabidopsis thaliana, and our current understanding of their roles for the regulation of metal import in plant cells.

\section{The specifics of the plant endocytic pathways}

Several endocytic pathways have been described in plants (Paez Valencia et al. 2016), driven by the specific recruitment of proteins at various location along the endocytic pathway. The most prominent one is the clathrin-mediated endocytosis (CME), where membrane invaginations with high concentrations of proteins to be transported (collectively referred to as cargo proteins or just cargo) are formed at the plasma membrane (Kaksonen and Roux 2018). Adaptor proteins, such as the heterotetrameric ADAPTOR PROTEIN2 (AP2) protein complex, bind specific sequences on the cargo, the membrane and clathrin heavy and light chain proteins. The result is the formation of clathrin-coated vesicles (Reynolds et al. 2018), which are then targeted towards endosomal compartments. Experiments tracing the endocytosis of the lipophilic dye FM4-64 show that disturbing CME using a dominant-negative form of clathrin drastically reduces the overall internalization of plasma membrane material (Dhonukshe et al. 
2007), suggesting that this might be the predominant endocytic pathway in plant cells. CME was found to function in all cells in the Arabidopsis root (Baral et al. 2015), thus indicating its significance to global plant physiology. Surprisingly, and in contrast to mammalian systems, AP2 appears to be dispensable for plant endocytosis and plant development (Fan et al. 2013; Yamaoka et al. 2013), suggesting the existence of other adaptors in plants. The TPLATE complex (TPC) has been proposed to serve as such alternative adaptor (Gadeyne et al. 2014). TPC is recruited as dynamic foci at the plasma membrane prior to the recruitment of AP2, clathrin, and dynamin-related proteins. TPC also shows non-overlapping profiles to AP2, pointing to specific roles in plant endocytosis. TPC is absolutely essential to both plant development and endocytosis, as evidenced by the lethal phenotype of mutants of TPC subunits (Gadeyne et al. 2014). Other interesting features of plant endocytosis are that plant clathrincoated vesicles are formed faster and differ in size to those in yeast and some mammalian systems, their formation does not require actin, and have a delayed uncoating process (Narasimhan et al. 2020). Alternatives to CME also exist in plants. A clathrin-independent pathway, targeting membrane-anchored but not transmembrane proteins was described in root epidermis cells under standard growth conditions (Baral et al. 2015). At the same time, salt stress was found to induce another clathrin-independent pathway for transmembrane and membrane-asociated (GPI-anchored) proteins that was dependent on the ADP-Ribosylation Factor-guanidine exchange factor (ARF-GEF) VACUOLAR PROTEIN SORTING6 (Baral et al. 2015), consistent with the fact that the clathrin-independent endocytosis of the transmembrane aquaporin PLASMA MEMBRANE INTRINSIC PROTEIN2;1 (PIP2;1) was enhanced under salt stress ( $\mathrm{Li}$ et al. 2011). Thus, the type of endocytosis a plant protein will undergo is not fixed and may depend on a variety of factors, such as the position of the cell, its developmental state and the signals/stimuli from environmental factors. The Arabidopsis FLOTILLIN1 (FLOT1) protein, similar to its mammalian homologs, was found to associate with detergent-resistant membrane microdomains and to participate in clathrin-independent 
formation of endocytic vesicles (Borner et al. 2005; Li et al. 2012). The brassinosteroidmediated activation of the BRASSINOSTEROID INSENSITIVE1 (BRI1) receptor kinase was shown to increase the colocalization between BRI1 and FLOT1, and promote the microdomainassociated BRI1 endocytosis (Wang et al. 2015).

Over the past decades, ubiquitin emerged as a crucial signal to remove plant plasma membrane proteins from the cell surface by endocytosis and to degrade them in the vacuole (Traub 2009). In yeast and mammals, ubiquitin is recognized by the ubiquitin-binding adaptor proteins Epsin/Eps15-like (Traub 2009). These adaptors are able to associate with clathrin, AP2, and phosphatidylinositol 4,5-bisphosphate PI(4,5)P2 lipid heads using their Epsin N-terminal homology (ENTH) domain. Considering that plant ENTH-like proteins do not possess conserved ubiquitin-binding domains (Song et al. 2012), it is very likely that plants use specific and yet to be characterized adaptors for ubiquitinated cargos internalization.

Following removal from the plasma membrane, the cargo enters the early endosome. Plants do not have a dedicated early endosome but this function is taken over by the trans-Golgi Network (TGN, hereafter called TGN/EE) (Dettmer et al. 2006), where recycling of plasma membrane proteins and sorting receptors occurs (Kunzl et al. 2016; Robinson and Neuhaus 2016; Heucken and Ivanov 2018). Interestingly, a recent report showed that these features of the TGN might be shared with Saccharomyces cerevisiae (Day et al. 2018). The TGN/EE of plants is devoid of phosphatidylinositol 3-phosphate (PI3P) which rather accumulates in multivesicular bodies (MVB)/late endosomes (LE) and at the vacuolar membrane (Simon et al., 2014). The role of endosomes in plant protein trafficking was first demonstrated by studying the effect of the ARFGEF GNOM. GNOM was found to be a target of the fungal drug Brefeldin A (BFA) and to be essential for the recycling of the auxin transporter PIN-FORMED1 (PIN1) at endosomes (Geldner et al. 2003). BFA treatment caused the overaccumulation of PIN1 in aberrant endosomal compartments in a GNOM-dependent manner and prevented PIN1 recycling. Other known factors involved in the sorting and recycling of endocytosed cargo in plants include 
sorting nexins (SNX), core retromer components, and the FYVE1/FREE1 protein (Jaillais et al. 2006; Jaillais et al. 2007; Zelazny et al. 2013; Barberon et al. 2014). If endocytosed proteins are targeted for degradation, they are sorted with the help of the large and sequentially-formed Endosomal Sorting Complexes Required for Transport (ESCRT) complex and are internalized in intraluminal vesicles of the MVB/LE. While the pathway shares many common principles with mammals and yeast, in plants it has several unique features. Plants apparently lack ESCRT-0 and a subunit of ESCRT-I (Leung et al. 2008), however this absence is compensated by the plant-specific TOM1-LIKE (TOL) proteins as well as the SRC HOMOLOGY-3 (SH3) DOMAIN-CONTAINING PROTEIN2 (SH3P2) (Korbei et al. 2013; Nagel et al. 2017). TOL proteins possess all functionally relevant features for the recognition and the sorting of ubiquitinated cargos, including i) the ability to bind K63 polyubiquitin chains and clathrin, ii) the localization to TGN/EE, and iii) the ability to associate with ESCRT-I subunits (Korbei et al. 2013; Moulinier-Anzola et al. 2020). Interestingly, TOLs show a broad range of localization along the endocytic pathway with TOL6 being found at the plasma membrane (MoulinierAnzola et al. 2020), suggesting that the sorting of ubiquitinated cargos is initiated at or close to the cell surface. This is however in disagreement with the observation that ubiquitinated cargos such as PIN2 are still sensitive to aggregation by BFA in a tol mutant background when de novo protein synthesis is blocked (Korbei et al. 2013). One possibility is that TOLs like TOL6 associate with ubiquitinated cargos at the plasma membrane with the actual sorting process occurring only later in endosomes. Alternatively, TOL6 may act at the cell surface, compensating for the lack of plant ENTH-like proteins able to bind ubiquitin, but cargos like PIN2 also have ubiquitin-independent internalization routes. Similarly, SH3P2 binds K63 polyubiquitinated proteins and is found at the plasma membrane, in clathrin-coated vesicles and in endosomes (Nagel et al. 2017). SH3P2 interacts with clathrin and some of the plant ESCRTI subunits likely to direct ubiquitinated cargos into the ESCRT pathway. Other plant-specific proteins in this complex include the ESCRT-III subunit FYVE1/FREE1 involved in 
phytohormone signaling and nutrient response (Barberon et al. 2014; Gao et al. 2014; BeldaPalazon et al. 2016; Xia et al. 2020; Xiao et al. 2020). Thus, analogy with yeast and mammalian systems might serve as clue for plant endocytosis and endomembrane trafficking, however detailed understanding of regulatory events in nutrient acquisition requires sophisticated experimentation in plant cells.

\section{Plant metal transporters are endocytic cargos}

The precise control in time and space of the abundance of cell surface transporters plays a fundamental role in keeping in check what comes in and out of the cell. This is all the more important to plants that lack cognition and motility, and thus cannot relocate to more favorable environment conditions. The removal of transporters from the cell surface by endocytosis and their possible recycling or targeting to the vacuole for degradation therefore constitute crucial mechanisms for homeostatic control (Zelazny and Vert 2014). A wealth of information was gathered over the past decade on how the dynamics of plant nutrient transporters is regulated and how that contributes to a fine-tuning of plant responses to nutrient shortage or excess (Zelazny and Vert 2014). Pioneering work using metal transporters from the model plant Arabidopsis thaliana shed light on the intricate network of signals and factors driving their dynamics and further highlighted the complexities and originalities of the plant endocytic pathway.

Metals are taken up by several families of metal transporters in plants, two of which have been shown to be controlled by endocytosis. The ZRT/IRT-like Protein (ZIP) family of transporters are membrane-localized transport proteins driving the influx of metals in the cytoplasm (Guerinot 2000). Most of the knowledge gained on plant ZIPs arose from the characterization of the Arabidopsis IRON-REGULATED TRANSPORTER1 (IRT1) transporter, the founding member of the ZIP family. IRT1 transports a wide variety of divalent metals including $\mathrm{Fe}, \mathrm{Zn}, \mathrm{Mn}, \mathrm{Co}, \mathrm{Cd}$ and probably others in root epidermal cells (Eide et al. 
1996; Korshunova et al. 1999; Rogers et al. 2000; Vert et al. 2001; Vert et al. 2002). Despite such low selectivity for metals, the primary metal substrate of IRT1 appears to be Fe since i) IRT1 is specifically expressed under low Fe conditions only (Eide et al. 1996; Vert et al. 2002), and ii) the irtl knock-out mutant that is severely chlorotic is reverted back to wild type-like appearance upon application of iron only (Varotto et al. 2002; Vert et al. 2002). IRT1 therefore represents the major entry route for $\mathrm{Fe}$ in plants, and also mediates the unwanted acquisition of highly reactive $\mathrm{Mn}, \mathrm{Zn}, \mathrm{Co}$ and $\mathrm{Cd}$ ions that tend to overaccumulate in plants tissues upon $\mathrm{Fe}$ deficiency (Vert et al. 2002; Barberon et al. 2011). The second family of plant metal transporters subjected to endocytosis is NATURAL RESISTANCE ASSOCIATED MACROPHAGE PROTEIN (NRAMP). NRAMP transporters also show low selectivity and are mostly involved in Fe and Mn transport in plant cells (Curie et al. 2000; Thomine et al. 2000; Cailliatte et al. 2010; Lanquar et al. 2010; Castaings et al. 2016; Alejandro et al. 2017; Li et al. 2019). NRAMPs harbor diverse subcellular distribution underlining their site of action. NRAMP1 is found in part at the cell surface and contributes, together with IRT1, to Fe and Mn uptake while NRAMP3 and NRAMP4 are localized to the vacuolar membrane and drive the remobilization of Fe and Mn from the vacuole (Thomine et al. 2003; Cailliatte et al. 2010; Lanquar et al. 2010; Agorio et al. 2017). Consistently, nramp knock-out mutants display several defects, including chlorosis and reduced growth, resulting from limited metal availability in the cytosol.

Although involved in the provision of enough metals to cells to sustain biochemical processes and growth, metal transporters should be rapidly switched off when cells face metal excess. The regulation of transporter abundance or localization thus appear as a crucial mechanism to limit the overaccumulation of metals and avoid subsequent toxicity. Early work on IRT1 highlighted its dual localization to the outer plasma membrane domain of root epidermal cells, facing the rhizosphere, and to intracellular structure co-localizing with the Vhaa1 subunit of the v-ATPase that serves as a TGN/EE marker in plants (Figure 2) (Barberon et al. 2011; Barberon et al. 2014). Consistent with the IRT1 presence in the TGN/EE, it 
accumulates in large aberrant compartments upon BFA treatment that aggregates the endosomal compartments (Barberon et al. 2011; Dubeaux et al. 2018). More detailed co-localization analyses revealed that IRT1 localization spans the endocytic pathway with also a small fraction of IRT1 colocalizing with the RabF2a LE marker (Dubeaux et al. 2018). Besides, IRT1 was also shown to reach the vacuolar lumen upon inhibition of the vacuolar lytic activity in darkness (Barberon et al. 2011), indicating that IRT1 follows the endocytic route in resting condition. Similarly, the Arabidopsis NRAMP1 metal transporter is mainly found in vesicular structures colocalizing with markers of the trans-Golgi (ST), TGN/EE (SYP43), and MVB/LE (ARA7, ARA6) and at the plasma membrane (Agorio et al. 2017). NRAMP1 steady-state localization pattern is dependent on endocytosis. An NRAMP1-GFP fusion was shown to accumulate in cytosolic compartments upon BFA treatment and then to relocate to the plasma membrane upon BFA washout (Gao et al. 2017). Pharmacological inhibition of the vacuolar ATPase using concanamycin A, which impairs vacuolar protease activity also revealed that NRAMP1 reaches the vacuole to be turned over. The NRAMP1 homolog, NRAMP2, is also found partially in the Golgi, colocalizing with the sialyltranferase marker, and in the TGN/EE where it perfectly overlaps with the SYP61 TGN/EE marker (Gao et al. 2018). Whether NRAMP2 is a TGN/EE resident protein or whether it traffics to the plasma membrane or the vacuole under certain conditions remains to be determined. Altogether, these observations were the starting point of establishing IRT1 and, to a lesser extent, NRAMP1 as model plant plasma membrane proteins to investigate deeper plant endocytosis and its relevance to plant physiology.

\section{CME and ubiquitin regulate metal transporter internalization and plant metal nutrition}

The internalization of IRT1 from the cell surface requires its ubiquitination and its recognition by specific adaptors. Early evidence using Tyrphostin A23 pointed to the requirement of AP2 adaptors for IRT1 endocytosis (Barberon et al. 2011), but this drug was 
recently shown to act as a protonophore in plants and thus only indirectly impacting endocytosis (Dejonghe et al. 2016). The involvement of AP2 must therefore been tested genetically, as already done for a number of plant cargos such as the brassinosteroid receptor BRI1 (Di Rubbo et al. 2013), or the PIN auxin efflux carriers (Fan et al. 2013; Kim et al. 2013). Since AP2 appears dispensable for endocytosis in plants and that the TPC adaptor complex show overlapping, but also distinct, functions with AP2 that are crucial to plant endocytosis (Gadeyne et al. 2014), it would be also interesting to evaluate whether TPC also contributes to IRT1 internalization.

The signal triggering IRT1 internalization from the plasma membrane is the monoubiquitination of two lysine residues, K154 and K179, in the cytoplasmically-exposed stretch, named variable region (vr), a characteristic of ZIP transporters (Figure 2A) (Barberon et al. 2011). Mutating both lysines makes the protein non-ubiquitinatable and causes its overaccumulation at the plasma membrane. Furthermore, ubiquitination-defective IRT1 is not sensitive to the aggregating effect of BFA, indicating that ubiquitination of IRT1 is crucial to drive IRT1 internalization from the cell surface (Dubeaux et al. 2018). This is in contrast to most plant cargos studied so far where ubiquitin mostly acts in endosomal sorting (Kasai et al. 2011; Leitner et al. 2012; Martins et al. 2015). IRT1 therefore represents an excellent model to dissect the roles of ubiquitination in cargo internalization and discriminate whether TOLs or SH3P2 act directly at the cell surface and/or later in endosomes.

Plants expressing the non-ubiquitinatable IRT1 protein suffer from excessive metal uptake, causing oxidative damage, which could eventually have a lethal outcome (Barberon et al. 2011). This fact underscores the crucial role of IRT1 endocytosis for maintenance of iron homeostasis and plant survival (Barberon et al. 2011). Ubiquitination of IRT1 is likely mediated by the RING-type E3 ubiquitin ligase IRT1 DEGRADATION FACTOR1 (IDF1). IDF1 was identified in a mutant screen for abnormally increased IRT1 accumulation (Figure 2A) (Shin et al. 2013). In the absence of IDF1, plants can better tolerate Fe deficiency, however due to the 
broad IRT1 substrate range, other metals, such as Mn, Zn will be imported into the cell with increased efficiency.

While IRT1 gene expression is affected by Fe availability, the IRT1 protein has a steady turnover rate not affected by Fe levels (Barberon et al. 2011; Dubeaux et al. 2018). Surprisingly, IRT1 protein stability is under the control of its non-Fe metal substrates such as $\mathrm{Zn}$ or Mn (Dubeaux et al. 2018). When plants face low Zn or Mn levels in soils, IRT1 is at the cell surface to take up Fe. Increasing $\mathrm{Zn}$ or Mn concentrations lead to the localization of IRT1 to both the plasma membrane and TGN/EE, likely as a result of its multiple monoubiquitination (Barberon et al. 2011; Dubeaux et al. 2018). Plants challenged with $\mathrm{Zn}$ or Mn excess target IRT1 to vacuolar degradation through the IDF1-mediated conversion of monoubiquitin to K63-linked polyubiquitin chains at residues K154 and K179 by IDF1 (Figure 2B) (Dubeaux et al. 2018). A characteristic of the ZIP transporters is a histidine-rich metal coordination site in the variable region, which in IRT1 is able to coordinate, among others, Mn and Zn (Guerinot 2000; Grossoehme et al. 2006; Dubeaux et al. 2018). While this does not affect IRT1-mediated transport, binding of $\mathrm{Mn}$ and $\mathrm{Zn}$ to such motif reduces the IRT1 lifetime by causing premature receptor degradation (Dubeaux et al. 2018). Coordination of Mn or $\mathrm{Zn}$ makes the variable region of IRT1 (IRT1vr) a target of the calcium-dependent protein kinase CBLINTERACTING PROTEIN KINASE23 (CIPK23). IRT1 phosphorylation triggers the dissociation of the high affinity iron uptake complex between IRT1, the $\mathrm{H}^{+}$-ATPase AHA2 and the reductase FERRIC REDUCTION OXIGENASE2 FRO2 (Martin Barranco et al. 2020). The phosphorylated IRT1vr is then recognized by IDF1, resulting in the K63 ubiquitination of IRT1, its relocalization to the LE and subsequent degradation (Dubeaux et al. 2018). This effect is particularly prominent under combined Fe deficiency and excess of the non-Fe IRT1 substrates, as the transporter is abundant and active due to the low availability of $\mathrm{Fe}$ (Figure 2B). Triggering this degradation mechanism prevents the import of toxic amounts of Mn and $\mathrm{Zn}$. Consistently, under such conditions cipk23 and idf1 mutants, as well as plants expressing a mutated IRT1 
lacking metal coordinating capacity, show significantly increased metal content in comparison with control plants (Dubeaux et al. 2018). It should be noted that the event initiating IRT1 endocytosis in this case is the metal binding by the variable region. Thus, IRT1 acts as both a transporter as well as receptor for these metals, and serves as a scaffold for the signaling events leading to its own degradation (Cointry and Vert 2019). Such types of transporter proteins are designated as transceptors and have been found in a variety of distantly-related organisms. In plants, such protein is for example the nitrate transporter NITRATE TRANSPORTER1.1 (NRT1.1) (Ho et al. 2009), however notably several ZIP-family transporters in yeast and mammals have also been shown to function as sensors. For example, the human $\mathrm{Zn}$ transceptor hZIP4 was shown to sense its substrate and change the outcome of its endocytosis. Under physiological zinc provision, zinc binding to hZIP4 transport site initiates a zinc-dependent constitutive endocytosis in a ubiquitin-independent manner that do not lead to hZIP4 degradation (Zhang et al. 2020). Upon Zn excess, elevated cytosolic $\mathrm{Zn}$ is sensed by the histidine-rich stretch in hZIP4 variable region, switching the endocytic trafficking mode of hZIP4 from endosomal recycling and retargeting to the plasma membrane to the targeting to LE and the lysosome for degradation (Mao et al. 2007; Zhang et al. 2020). In yeast, endocytosis of plasma membrane-localized ZIP transporter ZRT1 is dependent on the Zn supply. Under high Zn, ZRT1 is ubiquitinated at position K195 within its variable region and sent for vacuolar degradation (Gitan and Eide 2000). This coincides with the activation of the protein kinase a (PKA) signaling pathway, which happens only in the presence of ZRT1, suggesting that it may function as a receptor (Schothorst et al. 2017).

At present, it is unclear whether CME is the only mode of IRT1 endocytosis, and data on a recently-described IRT1-interacting protein may suggest the existence of alternative pathways. The $\mathrm{C} 2$ domain-containing small peripheral membrane protein ENHANCED BENDING1 (EHB1) was recently identified as an IRT1 inhibitor (Khan et al. 2019) (Figure 2A). In the presence of calcium, EHB1 associates to phosphatidylinositol (PI) and 
phosphatidylinositol 4-phosphate (PI4P) lipid heads on the plasma membrane cytoplasmic side. This is followed by a second interaction with neighboring lipids, which exposes its CARsignature domain for interaction the two amino acid stretches within the IRT1vr. The result of the EHB1-IRT1 interaction is the rapid, yet reversible, inhibition of IRT1 and overall reduction of Fe acquisition in plants occurring in response to calcium signaling (Khan et al. 2019) (Figure 2A), and supported by the fact that calcium levels in the root are elevated under Fe deficiency (Gratz et al. 2019). In addition to this, indirect evidence suggests that EHB1 might participate in events related to IRT1 endocytosis. In ehb1 mutants, IRT1 protein abundance is increased, suggesting that EHB1 might negatively affect IRT1 stability. At the same time, EHB1 homologs from the CAR protein family were shown to cause tubulation of liposome membranes in vitro (Diaz et al. 2016). Hypothetically, EHB1 might contribute to IRT1-containing vesicle formation at the plasma membrane, thus promoting its endocytosis and degradation, however further research will be needed to confirm or disprove such a possibility.

Besides IRT1, very little is known about the mechanisms of NRAMP1 or other metal transporter internalization.

\section{Sorting, recycling and vacuolar degradation of endocytosed metal transporters}

Once endocytosed, ubiquitinated proteins are recognized by the ESCRT complex and sorted into LE to be targeted to the vacuole. A plant-specific subunit of the ESCRT-I complex was directly linked to IRT1 endocytosis. The Arabidopsis FYVE1/FREE1 protein was indeed shown to interact with IRT1 and to be recruited to LE through phosphatidylinositol 3-phosphate (PI3P) binding (Barberon et al. 2014). Most importantly, FYVE1/FREE1 binds ubiquitin and several ESCRT-I subunits including ELC/AtVPS23A and AtVPS23B (Barberon et al. 2014; Gao et al. 2014). Modulation of FYVE1/FREE1 gene expression in transgenic plants not only alters global ubiquitinated protein accumulation in the membrane fraction, but also specifically impacts the trafficking of IRT1 to the vacuole (Barberon et al. 2014). Plants overexpressing 
FYVE1/FREE1 notably show increased accumulation of IRT1 at the cell surface and a loss of its lateral polarity (Barberon et al. 2014). The inability of IRT1 to reach the vacuole when FYVE1/FREE1 expression is modified likely results in aberrant recycling of IRT1 outside of its original plasma membrane domain. Such loss of polarity leads to a defect of radial transport of metals from root epidermal cells to central vascular tissues, highlighting the critical role of proper endosomal sorting for IRT1 (Barberon et al. 2014). FYVE1/FREE1 is subjected to degradation upon low iron through the interplay between SINAT1-4 RING E3 ligases and their inactive SINAT5 counterpart (Xiao et al. 2020), to fine tune the levels and localization of IRT1. Another Arabidopsis LE-localized PI3P-binding protein, PH1, was also demonstrated to control the vacuolar targeting of NRAMP1 (Agorio et al. 2017). Mutants lacking PH1 function mislocalize NRAMP1 to the vacuolar membrane instead of inside the vacuolar lumen due to defective endosomal sorting. Surprisingly, mislocalized NRAMP1 appears to be active and mediates the remobilization of metals from the vacuole, thus suppressing the iron deficiency phenotype of the nramp3nramp4 mutant (Agorio et al. 2017). In contrast to fyvel/freel mutant, which is lethal and has major vacuolar biogenesis defects, phl mutants are perfectly viable suggesting that PH1 likely affects the trafficking of a limited number of cargos. Further downstream along the ESCRT pathway, the ESCRT-II and -III complexes drive the formation and scission of intraluminal vesicles and the deubiquitination of cargo proteins. Cargo deubiquitination in plants is achieved by the LE-localized ASSOCIATED MOLECULE WITH THE SH3 DOMAIN OF STAM3 (AMSH3) deubiquitinase that interacts with several ESCRTIII subunits and shows activity towards K63-linked polyubiquitin chains (Isono et al. 2010; Katsiarimpa et al. 2011; Katsiarimpa et al. 2014). Loss of AMSH3 indeed affects the formation of intraluminal vesicles in LE, vacuolar biogenesis and the trafficking of cargo proteins towards the vacuole (Isono et al. 2010). Several subunits of the ESCRT-III complex have been characterized in plants (Katsiarimpa et al. 2014; Cardona-Lopez et al. 2015; Kalinowska et al. 2015), but their importance for IRT1 or NRAMP1 trafficking has not been reported yet. 
Regardless, both metal transporters are likely clients of the ESCRT pathway and are targeted to the vacuolar lumen for degradation.

Ubiquitinated cargos can be recycled through deubiquitination before intraluminal vesicle scission and therefore escape vacuolar degradation. To date, no plant deubiquitinase has been directly associated with deubiquitination of cargo for recycling (Isono and Nagel 2014). However, AMSH3 also colocalizes with clathrin and associates with SH3P2 (Nagel et al. 2017), suggesting that AtAMSH3 may also act early in the pathway to mediate the deubiquitination and recycling of endocytosed cargos. IRT1 is recycled using the endosomal regulatory SNX1 protein, which in plants has developed separate functions from the retromer complex (Pourcher et al. 2010; Ivanov et al. 2014; Ivanov and Robinson 2020). Both IRT1 and SNX1 partly colocalize in TGN/EE, and to a lesser extent in LE, and the loss of SNX1 activity in snx 1 mutants yield lower IRT1 protein accumulation and increased degradation (Ivanov et al. 2014). This is also highlighted by the reduced IRT1 protein accumulation at the cell surface in snxl, indicating that IRT1 recycling requires the action of SNX proteins to avoid premature degradation. Consistently, snxl knock-out and higher order snx mutants are hypersensitive to iron deficiency, take up less iron from the soil, and show altered expression of iron-regulated genes (Blum et al. 2014; Ivanov et al. 2014). Transporter recycling might also be under environmental and developmental regulation, as SNX1 and its two homologs SNX2a, and SNX2b undergo phosphorylation, which in SNX1 depends on auxin signaling and ionizing radiation (Zhang et al. 2013; Roitinger et al. 2015; Brumbarova and Ivanov 2016). Furthermore, choline concentrations were shown to influence the transporter recycling process. The absence of the CHOLINE TRANSPORTER-LIKE1 (CTL1) protein compromises the recycling of NRAMP1, an effect, which can be mimicked by modulating choline concentration externally (Gao et al. 2017). CTL1 localizes at the TGN/EE, LE and the plasma membrane, and affects the recycling of other transporters, such as the auxin transporters PIN1 and PIN3 (Wang et al. 2017). The nature of the choline effect on recycling is not yet clear, however the modified lipid 
contents in CTL1 loss-of-function plants suggest that choline might influence the composition and nature of the endosomal surface (Gao et al. 2017; Wang et al. 2017).

Besides ubiquitin-mediated endocytosis, the MxIRT1 ortholog of IRT1 from apple has been proposed to undergo ubiquitin-mediated autophagic degradation upon iron excess when expressed in yeast ( $\mathrm{Li}$ et al. 2015). Although interesting, further demonstration that such mechanism occurs in plants will be needed.

\section{Conclusions}

In the last decade, our knowledge about plant endocytosis has made a major leap forward. This has been made possible by i) the detailed study of endocytosis-related proteins, based on homology with what is known in yeast and mammals, ii) the analysis of plant cargo endocytosis including IRT1, and iii) the identification of new endocytic factors or regulatory mechanisms through clever genetic screens or biochemical approaches searching for interacting proteins. Most importantly, the joint efforts from the plant endocytosis and the plant transporter/receptor scientific communities shed light on the fundamental roles played by endocytosis in the deactivation or the degradation of plasma membrane proteins, or in signaling. In addition, working with plants has the great potential to decipher the contribution of plant endocytosis and plasma membrane protein dynamics to essential biological function at the level of a multicellular organism. Working with plant metal transporters notably helped to untangle the intricate mechanisms of ubiquitin-mediated endocytosis and sorting and revealed the importance of keeping transporter levels and localization in check to maintain proper metal homeostasis.

Recent years have seen the ever wider use of advanced cross-disciplinary methods and the ever increasing spatial and temporal resolution of imaging techniques. The use of total internal reflection fluorescence (TIRF) microscopy, Förster resonance energy transfer analysis using florescence lifetime imaging (FRET FLIM), or super resolution microscopy in plants now 
allow plant scientist to push further the limit of what can be observed in plant cells. With such a powerful arsenal in hands, many open questions concerning the mechanisms of plant endomembrane trafficking and their role in metal homeostasis will find explanations, opening up new research horizons.

\section{Acknowledgements}

We thank members of the Vert lab for helpful suggestions. Work in the Vert lab is supported by the Agence Nationale de la Recherche (grant ANR-13-JSV2-0004-01 to G.V.), the French Laboratory of Excellence project "TULIP" (ANR-10-LABX-41 and ANR-11-IDEX-0002-02 to G.V.).

Agorio A, Giraudat J, Bianchi MW, Marion J, Espagne C, Castaings L, Lelièvre F, Curie C, Thomine S, Merlot S. 2017. Phosphatidylinositol 3-phosphate-binding protein AtPH1 controls the localization of the metal transporter NRAMP1 in Arabidopsis. Proc Nat Acad Sci 114, E3354-E3363.

Alejandro S, Cailliatte R, Alcon C, Dirick L, Domergue F, Correia D, Castaings L, Briat JF, Mari S, Curie C. 2017. Intracellular Distribution of Manganese by the Trans-Golgi Network Transporter NRAMP2 Is Critical for Photosynthesis and Cellular Redox Homeostasis. Plant Cell 29, 3068-3084.

Baral A, Irani NG, Fujimoto M, Nakano A, Mayor S, Mathew MK. 2015. Salt-induced remodeling of spatially restricted clathrin-independent endocytic pathways in Arabidopsis root. Plant Cell 27, 1297-1315.

Barberon M, Dubeaux G, Kolb C, Isono E, Zelazny E, Vert G. 2014. Polarization of IRONREGULATED TRANSPORTER 1 (IRT1) to the plant-soil interface plays crucial role in metal homeostasis. Proc Nat Acad Sci 111, 8293-8298.

Barberon M, Zelazny E, Robert S, Conejero G, Curie C, Friml J, Vert G. 2011. Monoubiquitindependent endocytosis of the iron-regulated transporter 1 (IRT1) transporter controls iron uptake in plants. Proc Nat Acad Sci 108, E450-458.

Belda-Palazon B, Rodriguez L, Fernandez MA, Castillo MC, Anderson EA, Gao C, GonzalezGuzman M, Peirats-Llobet M, Zhao Q, De Winne N et al. 2016. FYVE1/FREE1 Interacts with the PYL4 ABA Receptor and Mediates its Delivery to the Vacuolar Degradation Pathway. Plant Cell 24, 2291-2311.

Blum A, Brumbarova T, Bauer P, Ivanov R. 2014. Hormone influence on the spatial regulation of IRT1 expression in iron-deficient Arabidopsis thaliana roots. Plant Signal Behav 9, e28787.

Borner GH, Sherrier DJ, Weimar T, Michaelson LV, Hawkins ND, Macaskill A, Napier JA, Beale MH, Lilley KS, Dupree P. 2005. Analysis of detergent-resistant membranes in Arabidopsis. Evidence for plasma membrane lipid rafts. Plant Physiol 137, 104-116. 
Brumbarova T, Ivanov R. 2016. Differential Gene Expression and Protein Phosphorylation as Factors Regulating the State of the Arabidopsis SNX1 Protein Complexes in Response to Environmental Stimuli. Front Plant Sci 7, 1456.

Cailliatte R, Schikora A, Briat JF, Mari S, Curie C. 2010. High-affinity manganese uptake by the metal transporter NRAMP1 is essential for Arabidopsis growth in low manganese conditions. Plant Cell 22, 904-917.

Cardona-Lopez X, Cuyas L, Marin E, Rajulu C, Irigoyen ML, Gil E, Puga MI, Bligny R, Nussaume L, Geldner N et al. 2015. ESCRT-III-Associated Protein ALIX Mediates High-Affinity Phosphate Transporter Trafficking to Maintain Phosphate Homeostasis in Arabidopsis. Plant Cell 27, 2560-2581.

Castaings L, Caquot A, Loubet S, Curie C. 2016. The high-affinity metal Transporters NRAMP1 and IRT1 Team up to Take up Iron under Sufficient Metal Provision. Sci Rep 6, 37222 .

Cointry V, Vert G. 2019. The bifunctional transporter-receptor IRT1 at the heart of metal sensing and signalling. New Phytologist 223, 1173-1178.

Curie C, Alonso JM, Le Jean M, Ecker JR, Briat JF. 2000. Involvement of NRAMP1 from Arabidopsis thaliana in iron transport. Biochem J 347, 749-755.

Day KJ, Casler JC, Glick BS. 2018. Budding Yeast Has a Minimal Endomembrane System. Dev Cell 44, 56-72 e54.

Dejonghe W, Kuenen S, Mylle E, Vasileva M, Keech O, Viotti C, Swerts J, Fendrych M, OrtizMorea FA, Mishev K et al. 2016. Mitochondrial uncouplers inhibit clathrin-mediated endocytosis largely through cytoplasmic acidification. Nat Commun 7, 11710.

Dettmer J, Hong-Hermesdorf A, Stierhof YD, Schumacher K. 2006. Vacuolar H+-ATPase activity is required for endocytic and secretory trafficking in Arabidopsis. Plant Cell 18, 715-730.

Dhonukshe P, Aniento F, Hwang I, Robinson DG, Mravec J, Stierhof YD, Friml J. 2007. Clathrin-mediated constitutive endocytosis of PIN auxin efflux carriers in Arabidopsis. Curr Biol 17, 520-527.

Di Rubbo S, Irani NG, Kim SY, Xu ZY, Gadeyne A, Dejonghe W, Vanhoutte I, Persiau G, Eeckhout D, Simon S et al. 2013. The clathrin adaptor complex AP-2 mediates endocytosis of brassinosteroid insensitivel in Arabidopsis. Plant Cell 25, 2986-2997.

Diaz M, Sanchez-Barrena MJ, Gonzalez-Rubio JM, Rodriguez L, Fernandez D, Antoni R, Yunta C, Belda-Palazon B, Gonzalez-Guzman M, Peirats-Llobet M et al. 2016. Calcium-dependent oligomerization of CAR proteins at cell membrane modulates ABA signaling. Proc Nat Acad Sci 113, E396-405.

Dubeaux G, Neveu J, Zelazny E, Vert G. 2018. Metal Sensing by the IRT1 TransporterReceptor Orchestrates Its Own Degradation and Plant Metal Nutrition. Mol Cell 69, 953-964 e955.

Eide D, Broderius M, Fett J, Guerinot ML. 1996. A novel iron-regulated metal transporter from plants identified by functional expression in yeast. Proc Nat Acad Sci 93, 5624-5628.

Fan L, Hao H, Xue Y, Zhang L, Song K, Ding Z, Botella MA, Wang H, Lin J. 2013. Dynamic analysis of Arabidopsis AP2 sigma subunit reveals a key role in clathrin-mediated endocytosis and plant development. Development 140, 3826-3837.

Gadeyne A, Sanchez-Rodriguez C, Vanneste S, Di Rubbo S, Zauber H, Vanneste K, Van Leene J, De Winne N, Eeckhout D, Persiau G et al. 2014. The TPLATE Adaptor Complex Drives Clathrin-Mediated Endocytosis in Plants. Cell 156, 691-704.

Gao C, Luo M, Zhao Q, Yang R, Cui Y, Zeng Y, Xia J, Jiang L. 2014. A unique plant ESCRT component, FREE1, regulates multivesicular body protein sorting and plant growth. Curr Biol 24, 2556-2563. 
Gao H, Xie W, Yang C, Xu J, Li J, Wang H, Chen X, Huang CF. 2018. NRAMP2, a transGolgi network-localized manganese transporter, is required for Arabidopsis root growth under manganese deficiency. New Phytol 217, 179-193.

Gao YQ, Chen JG, Chen ZR, An D, Lv QY, Han ML, Wang YL, Salt DE, Chao DY. 2017. A new vesicle trafficking regulator CTL1 plays a crucial role in ion homeostasis. PLoS Biol 15, e2002978.

Geldner N, Anders N, Wolters H, Keicher J, Kornberger W, Muller P, Delbarre A, Ueda T, Nakano A, Jurgens G. 2003. The Arabidopsis GNOM ARF-GEF mediates endosomal recycling, auxin transport, and auxin-dependent plant growth. Cell 112, 219-230.

Gitan RS, Eide DJ. 2000. Zinc-regulated ubiquitin conjugation signals endocytosis of the yeast ZRT1 zinc transporter. Biochem J 346, 329-336.

Gratz R, Manishankar P, Ivanov R, Koster P, Mohr I, Trofimov K, Steinhorst L, Meiser J, Mai HJ, Drerup M et al. 2019. CIPK11-Dependent Phosphorylation Modulates FIT Activity to Promote Arabidopsis Iron Acquisition in Response to Calcium Signaling. Dev Cell 48, 726-740 e710.

Grossoehme NE, Akilesh S, Guerinot ML, Wilcox DE. 2006. Metal-binding thermodynamics of the histidine-rich sequence from the metal-transport protein IRT1 of Arabidopsis thaliana. Inorganic Chemistry 45, 8500-8508.

Guerinot ML. 2000. The ZIP family of metal transporters. Biochim Biophys Acta 1465, 190198.

Heucken N, Ivanov R. 2018. The retromer, sorting nexins and the plant endomembrane protein trafficking. J Cell Sci 131, 1-9.

Ho CH, Lin SH, Hu HC, Tsay YF. 2009. CHL1 functions as a nitrate sensor in plants. Cell 138, 1184-1194.

Isono E, Katsiarimpa A, Muller IK, Anzenberger F, Stierhof YD, Geldner N, Chory J, Schwechheimer C. 2010. The deubiquitinating enzyme AMSH3 is required for intracellular trafficking and vacuole biogenesis in Arabidopsis thaliana. Plant Cell 22, 1826-1837.

Isono E, Nagel MK. 2014. Deubiquitylating enzymes and their emerging role in plant biology. Front Plant Sci 5, 56.

Ivanov R, Brumbarova T, Blum A, Jantke AM, Fink-Straube C, Bauer P. 2014. SORTING NEXIN1 is required for modulating the trafficking and stability of the Arabidopsis IRON-REGULATED TRANSPORTER1. Plant Cell 26, 1294-1307.

Ivanov R, Robinson DG. 2020. EMAC, Retromer, and VSRs: do they connect? Protoplasma.

Jaillais Y, Fobis-Loisy I, Miege C, Rollin C, Gaude T. 2006. AtSNX1 defines an endosome for auxin-carrier trafficking in Arabidopsis. Nature 443, 106-109.

Jaillais Y, Santambrogio M, Rozier F, Fobis-Loisy I, Miege C, Gaude T. 2007. The retromer protein VPS29 links cell polarity and organ initiation in plants. Cell 130, 1057-1070.

Kaksonen M, Roux A. 2018. Mechanisms of clathrin-mediated endocytosis. Nat Rev Mol Cell Biol 19, 313-326.

Kalinowska K, Nagel MK, Goodman K, Cuyas L, Anzenberger F, Alkofer A, Paz-Ares J, Braun $\mathrm{P}$, Rubio V, Otegui MS et al. 2015. Arabidopsis ALIX is required for the endosomal localization of the deubiquitinating enzyme AMSH3. Proc Nat Acad Sci 112, E55435551.

Kasai K, Takano J, Miwa K, Toyoda A, Fujiwara T. 2011. High boron-induced ubiquitination regulates vacuolar sorting of the BOR1 borate transporter in Arabidopsis thaliana. J Biol Chem 286, 6175-6183.

Katsiarimpa A, Anzenberger F, Schlager N, Neubert S, Hauser MT, Schwechheimer C, Isono E. 2011. The Arabidopsis deubiquitinating enzyme AMSH3 interacts with ESCRT-III subunits and regulates their localization. Plant Cell 23, 3026-3040. 
Katsiarimpa A, Munoz A, Kalinowska K, Uemura T, Rojo E, Isono E. 2014. The ESCRT-IIIinteracting deubiquitinating enzyme AMSH3 is essential for degradation of ubiquitinated membrane proteins in Arabidopsis thaliana. Plant \& Cell Physiology 55, 727-736.

Khan I, Gratz R, Denezhkin P, Schott-Verdugo SN, Angrand K, Genders L, Basgaran RM, Fink-Straube C, Brumbarova T, Gohlke H et al. 2019. Calcium-Promoted Interaction between the C2-Domain Protein EHB1 and Metal Transporter IRT1 Inhibits Arabidopsis Iron Acquisition. Plant Physiol 180, 1564-1581.

Kim SY, Xu ZY, Song K, Kim DH, Kang H, Reichardt I, Sohn EJ, Friml J, Juergens G, Hwang I. 2013. Adaptor protein complex 2-mediated endocytosis is crucial for male reproductive organ development in Arabidopsis. Plant Cell 25, 2970-2985.

Korbei B, Moulinier-Anzola J, De-Araujo L, Lucyshyn D, Retzer K, Khan MA, Luschnig C. 2013. Arabidopsis TOL proteins act as gatekeepers for vacuolar sorting of PIN2 plasma membrane protein. Curr Biol 23, 2500-2505.

Korshunova YO, Eide D, Clark WG, Guerinot ML, Pakrasi HB. 1999. The IRT1 protein from Arabidopsis thaliana is a metal transporter with a broad substrate range. Plant Mol Biol 40, 37-44.

Kunzl F, Fruholz S, Fassler F, Li B, Pimpl P. 2016. Receptor-mediated sorting of soluble vacuolar proteins ends at the trans-Golgi network/early endosome. Nat Plants 2, 16017.

Lanquar V, Ramos MS, Lelievre F, Barbier-Brygoo H, Krieger-Liszkay A, Kramer U, Thomine S. 2010. Export of vacuolar manganese by AtNRAMP3 and AtNRAMP4 is required for optimal photosynthesis and growth under manganese deficiency. Plant Physiol 152, 1986-1999.

Leitner J, Petrasek J, Tomanov K, Retzer K, Parezova M, Korbei B, Bachmair A, Zazimalova E, Luschnig C. 2012. Lysine63-linked ubiquitylation of PIN2 auxin carrier protein governs hormonally controlled adaptation of Arabidopsis root growth. Proc Nat Acad Sci 109, 8322-8327.

Leung KF, Dacks JB, Field MC. 2008. Evolution of the multivesicular body ESCRT machinery; retention across the eukaryotic lineage. Traffic 9, 1698-1716.

Li J, Wang Y, Zheng L, Li Y, Zhou X, Li J, Gu D, Xu E, Lu Y, Chen X et al. 2019. The Intracellular Transporter AtNRAMP6 Is Involved in Fe Homeostasis in Arabidopsis. Front Plant Sci 10, 1124.

Li R, Liu P, Wan Y, Chen T, Wang Q, Mettbach U, Baluska F, Samaj J, Fang X, Lucas WJ et al. 2012. A membrane microdomain-associated protein, Arabidopsis Flot1, is involved in a clathrin-independent endocytic pathway and is required for seedling development. Plant Cell 24, 2105-2122.

Li S, Zhang X, Zhang XY, Xiao W, Berry JO, Li P, Jin S, Tan S, Zhang P, Zhao WZ et al. 2015. Expression of Malus xiaojinensis IRT1 (MxIRT1) protein in transgenic yeast cells leads to degradation through autophagy in the presence of excessive iron. Yeast 32, 499517.

Li X, Wang X, Yang Y, Li R, He Q, Fang X, Luu DT, Maurel C, Lin J. 2011. Single-molecule analysis of PIP2;1 dynamics and partitioning reveals multiple modes of Arabidopsis plasma membrane aquaporin regulation. Plant Cell 23, 3780-3797.

Mao X, Kim BE, Wang F, Eide DJ, Petris MJ. 2007. A histidine-rich cluster mediates the ubiquitination and degradation of the human zinc transporter, hZIP4, and protects against zinc cytotoxicity. $J$ Biol Chem 282, 6992-7000.

Martin Barranco A, Spielmann J, Dubeaux G, Vert G, Zelazny E. 2020. Dynamic control of the high-affinity iron uptake complex in root epidermal cells. Plant Physiol doi: 10.1104/pp.20.00234.

Martins S, Dohmann EM, Cayrel A, Johnson A, Fischer W, Pojer F, Satiat-Jeunemaitre B, Jaillais Y, Chory J, Geldner $\mathrm{N}$ et al. 2015. Internalization and vacuolar targeting of the 
brassinosteroid hormone receptor BRI1 are regulated by ubiquitination. Nat Commun 6, 6151 .

Moulinier-Anzola J, Schwihla M, De-Araujo L, Artner C, Jorg L, Konstantinova N, Luschnig C, Korbei B. 2020. TOLs Function as Ubiquitin Receptors in the Early Steps of the ESCRT Pathway in Higher Plants. Molecular plant 13, 717-731.

Nagel MK, Kalinowska K, Vogel K, Reynolds GD, Wu Z, Anzenberger F, Ichikawa M, Tsutsumi C, Sato MH, Kuster B et al. 2017. Arabidopsis SH3P2 is an ubiquitin-binding protein that functions together with ESCRT-I and the deubiquitylating enzyme AMSH3. Proc Nat Acad Sci 114, E7197-E7204.

Narasimhan M, Johnson A, Prizak R, Kaufmann WA, Tan S, Casillas-Perez B, Friml J. 2020. Evolutionarily unique mechanistic framework of clathrin-mediated endocytosis in plants. Elife 9 doi:10.7554/eLife.52067.

Paez Valencia J, Goodman K, Otegui MS. 2016. Endocytosis and Endosomal Trafficking in Plants. Annual review of plant biology 67, 309-335.

Pourcher M, Santambrogio M, Thazar N, Thierry AM, Fobis-Loisy I, Miege C, Jaillais Y, Gaude T. 2010. Analyses of sorting nexins reveal distinct retromer-subcomplex functions in development and protein sorting in Arabidopsis thaliana. Plant Cell 22, 3980-3991.

Reynolds GD, Wang C, Pan J, Bednarek SY. 2018. Inroads into Internalization: Five Years of Endocytic Exploration. Plant Physiol 176, 208-218.

Robinson DG, Neuhaus JM. 2016. Receptor-mediated sorting of soluble vacuolar proteins: myths, facts, and a new model. $J$ Exp Bot 67, 4435-4449.

Rogers EE, Eide DJ, Guerinot ML. 2000. Altered selectivity in an Arabidopsis metal transporter. Proc Nat Acad Sci 97, 12356-12360.

Roitinger E, Hofer M, Kocher T, Pichler P, Novatchkova M, Yang J, Schlogelhofer P, Mechtler K. 2015. Quantitative phosphoproteomics of the ataxia telangiectasia-mutated (ATM) and ataxia telangiectasia-mutated and rad3-related (ATR) dependent DNA damage response in Arabidopsis thaliana. Mol Cell Proteomics 14, 556-571.

Schothorst J, Zeebroeck GV, Thevelein JM. 2017. Identification of Ftr1 and Zrt1 as iron and zinc micronutrient transceptors for activation of the PKA pathway in Saccharomyces cerevisiae. Microb Cell 4, 74-89.

Shin LJ, Lo JC, Chen GH, Callis J, Fu H, Yeh KC. 2013. IRT1 degradation factor1, a ring E3 ubiquitin ligase, regulates the degradation of iron-regulated transporter1 in Arabidopsis. Plant Cell 25, 3039-3051.

Simon MLA, Platre MP, Assil S, van Wijk R, Chen WY, Chory J, Dreux M, Munnik T, Jaillais Y. 2014. A multi-colour/multi-affinity marker set to visualize phosphoinositide dynamics in Arabidopsis. Plant $J$ 77, 322-337.

Song K, Jang M, Kim SY, Lee G, Lee GJ, Kim DH, Lee Y, Cho W, Hwang I. 2012. An A/ENTH domain-containing protein functions as an adaptor for clathrin-coated vesicles on the growing cell plate in Arabidopsis root cells. Plant Physiol 159, 1013-1025.

Thomine S, Lelièvre F, Debarbieux E, Schroeder JI, Barbier-Brygoo H. 2003. AtNRAMP3, a multispecific vacuolar metal transporter involved in plant responses to iron deficiency. Plant J 34, 685-695.

Thomine S, Vert G. 2013. Iron nutrition in plants : better be safe than sorry! Current opinion in plant biology 16, 322-327.

Thomine S, Wang R, Ward JM, Crawford NM, Schroeder JI. 2000. Cadmium and iron transport by members of a plant transporter gene family in Arabidopsis with homology to NRAMP genes. Proc Nat Acad Sci USA 97, 4991-4996.

Traub LM. 2009. Tickets to ride: selecting cargo for clathrin-regulated internalization. Nat Rev Mol Cell Biol 10, 583-596. 
Varotto C, Maiwald D, Pesaresi P, Jahns P, Salamini F, Leister D. 2002. The metal ion transporter IRT1 is necessary for iron homeostasis and efficient photosynthesis in Arabidopsis thaliana. Plant J 31, 589-599.

Vert G, Briat JF, Curie C. 2001. Arabidopsis IRT2 gene encodes a root-periphery iron transporter. Plant J 26, 181-189.

Vert G, Grotz N, Dedaldechamp F, Gaymard F, Guerinot ML, Briat JF, Curie C. 2002. IRT1, an Arabidopsis transporter essential for iron uptake from the soil and for plant growth. Plant Cell 14, 1223-1233.

Wang L, Li H, Lv X, Chen T, Li R, Xue Y, Jiang J, Jin B, Baluska F, Samaj J et al. 2015. Spatiotemporal Dynamics of the BRI1 Receptor and its Regulation by Membrane Microdomains in Living Arabidopsis Cells. Molecular plant 8, 1334-1349.

Wang Y, Yang L, Tang Y, Tang R, Jing Y, Zhang C, Zhang B, Li X, Cui Y, Zhang C et al. 2017. Arabidopsis choline transporter-like 1 (CTL1) regulates secretory trafficking of auxin transporters to control seedling growth. PLoS Biol 15, e2004310.

Xia FN, Zeng B, Liu HS, Qi H, Xie LJ, Yu LJ, Chen QF, Li JF, Chen YQ, Jiang L et al. 2020. SINAT E3 Ubiquitin Ligases Mediate FREE1 and VPS23A Degradation to Modulate Abscisic Acid Signalling. Plant Cell doi:10.1105/tpc.20.00267

Xiao Z, Yang C, Liu C, Yang L, Yang S, Zhou J, Li F, Jiang L, Xiao S, Gao C et al. 2020. SINAT E3 ligases regulate the stability of the ESCRT component FREE1 in response to iron deficiency in plants. J Integr Plant Biol 62, 1399-1417.

Yamaoka S, Shimono Y, Shirakawa M, Fukao Y, Kawase T, Hatsugai N, Tamura K, Shimada T, Hara-Nishimura I. 2013. Identification and dynamics of Arabidopsis adaptor protein2 complex and its involvement in floral organ development. Plant Cell 25, 2958-2969.

Zelazny E, Santambrogio M, Pourcher M, Chambrier P, Berne-Dedieu A, Fobis-Loisy I, Miege C, Jaillais Y, Gaude T. 2013. Mechanisms governing the endosomal membrane recruitment of the core retromer in Arabidopsis. J Biol Chem 288, 8815-8825.

Zelazny E, Vert G. 2014. Plant nutrition: root transporters on the move. Plant Physiol 166, 500508.

Zhang C, Sui D, Zhang T, Hu J. 2020. Molecular Basis of Zinc-Dependent Endocytosis of Human ZIP4 Transceptor. Cell Rep 31, 107582.

Zhang H, Zhou H, Berke L, Heck AJ, Mohammed S, Scheres B, Menke FL. 2013. Quantitative phosphoproteomics after auxin-stimulated lateral root induction identifies an SNX1 protein phosphorylation site required for growth. Mol Cell Proteomics 12, 1158-1169. 
Figure 1. Trafficking pathways for plasma membrane transporters in root epidermal cells. (A) Within a seedling, one of the functions of the root is the acquisition of nutrients, including metal ions from the soil. (B) In cross-section, the root of the model plant Arabidopsis includes several layers of cells. The outermost layer, named epidermis, is the main place where acquisition of nutrients occurs. (C) In epidermal cells, the availability of metal ion transporters at the plasma membrane is a result of complex trafficking events along the endomembrane system. Following synthesis at the endoplasmic reticulum (not depicted), transporters follow the secretion pathway (grey arrows) through the Golgi apparatus and the trans-Golgi Network to reach the plasma membrane. Plasma membrane transporters can be endocytosed (blue arrows) and sent back to the trans-Golgi Network, which in plant cells functions as an early endosome. At this point, transporter molecules can recycle back to the plasma membrane (green arrow), or be targeted for degradation (red arrows) in the lytic vacuole through the multivesicular body. Proteins and endomembrane structures in the figure are not drawn to scale.

Figure 2. Regulation of metal acquisition through endocytosis and endosomal recycling. (A) under low Fe, IRT1 is present at the plasma membrane and imports $\mathrm{Fe}^{2+}$ ions in the cytosol. Upon increase in external $\mathrm{Fe}$ concentrations, the accumulation of $\mathrm{Fe}^{2+}$ in the cytosol triggers Ca-mediated signaling events resulting in the inhibition of IRT1 through an interaction with EHB1. IRT1 internalization from the cell surface requires the multimonoubiquitination at residues K154 and K179, possibly mediated by the E3 ubiquitin ligase IDF1. At present, it is not clear whether EHB1 participates in the internalization process. (B) Under low Fe, the amount of the IRT1 secondary metal substrates such as $\mathrm{Zn}^{2+}$ and $\mathrm{Mn}^{2}$ may be high, potentially resulting in their excessive overaccumulation in the cytosol. IRT1 itself can coordinate $\mathrm{Zn}^{2+}$ and $\mathrm{Mn}^{2}$ using a histidine-rich stretch within its cytoplasmically-exposed variable region (upper insert). Metal binding to histidines allows the recruitment of the CIPK23 protein kinase and subsequent phosphorylation of serine and threonine residues within the variable region. These phosphosites serve as docking sites for IDF1, allowing the K63 polyubiquitination of two lysine residues, K154 and K179, and IRT1 targeting to the vacuole for degradation. (C) The fate of endocytosed IRT1 transporters depends on the covalent marks they have received and on several endocytic proteins driving the recycling or the endosomal sorting in the multivesicular body such as SNX1 and FYVE1/FREE1. (D) Polyubiquitinated IRT1 molecules entering the intraluminal vesicles are ultimately released in the vacuole for degradation. Note that IRT1 can thus both transport and sense its non-Fe substrates. At the same time, the NRAMP1 transporter found the multivesicular body and presumably coming from the plasma membrane, is 
internalized in the intraluminal vesicles with the help of the PH1 protein. Whether PH1 is involved in IRT1 internalization is not clear. Proteins and endomembrane structures in the figure are not drawn to scale. 


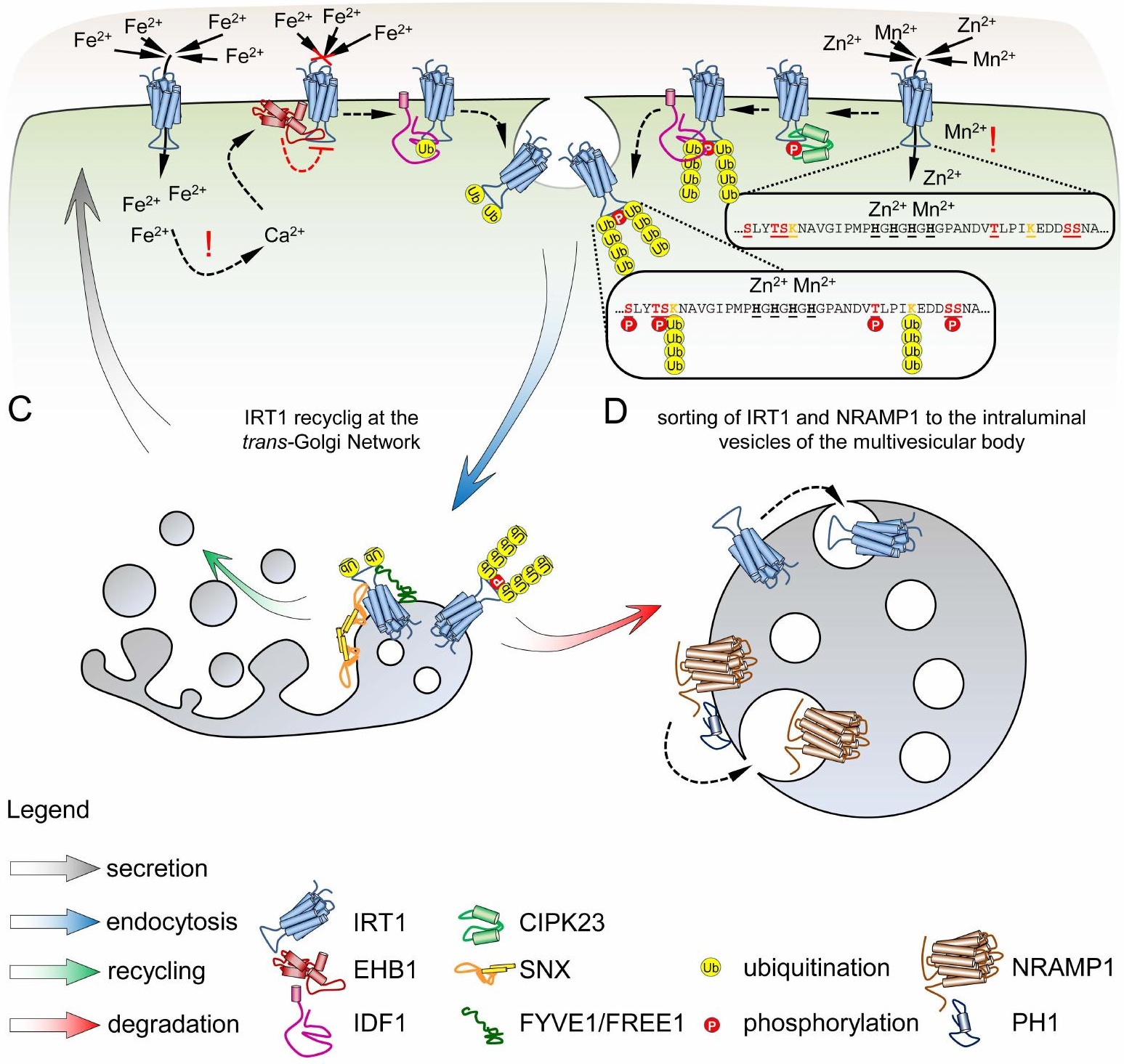

